\title{
Die Entwicklung von Geburtshaus-Standards für Europa
}

Rayment DJ, Rocca-Ihenacho DL, Newburn M, Thaels E, Laura B, McCourt PC. The development of Midwifery Unit Standards for Europe. Midwifery 2020; 86, doi: 10.1016/j.midw.2020.102661

\section{Zusammenfassung}

Erstmals wurden Standards für Geburtshäuser in einem aufwendigen Prozess entwickelt. Dieser umfasst eine systematische Literaturrecherche und Ergebnisse aus Verfahren, die die Ansichten von Praktikerinnen und Praktikern sowie Frauen, die Hebammenhilfe in Anspruch nehmen, mit Einschätzungen von Fachexpertinnen und -experten kombinieren. Durch die Transparenz des Prozesses könnten die Ergebnisse und das Vorgehen auch außerhalb Großbritanniens Anwendung finden. Das Vorgehen wird im Artikel genau beschrieben. Alle Ergebnisse sind in die "Midwifery Unit Standards" eingeflossen, die das Midwifery Unit Network und die City University of London 2018 publiziert haben [1].

\section{Ziele}

Bekannte Hebammenforscherinnen haben sich darangemacht, allgemeine Standards für Geburtshäuser zu entwickeln. Ziel war es, den Ratgeber über die Einrichtung, die Ausstattung und das Betreiben von Geburtshäusern (alleinstehend oder auf dem Klinikgelände) zu aktualisieren.

\section{Methodik}

Durch zwei Methoden sollte ein möglichst transparentes Verfahren erprobt werden: Zum einen wurde ein systematisches Literaturreview zur Frage nach den wichtigsten Hindernissen und fördernden Faktoren für gut funktionierende Geburtshäuser erstellt. Zum anderen haben die Forschenden ein sehr aufwendiges Befragungsverfahren durchgeführt.

Literaturreview: 24 Artikel und 3 Doktorarbeiten haben die Einschlusskriterien erfüllt und wurden nach den Kriterien von Walsh und Downe (2005) auf ihre Aussagkraft hin bewertet. Die Erkenntnisse aus der Forschungslage flossen in die Befragungen ein.

Befragungsverfahren: Für eine erste online Delphi Befragung wurden 122 Geburtshäuser aus 7 europäischen Ländern eingeladen, die bereits existierenden Standards des Royal College of Midwives zu bewerten und selbst Vorschläge zu machen. Etwa 50 Prozent der Angefragten nahmen teil. Ein erster Workshop wurde 2017 durchgeführt: 29 Akteurinnen und Akteure im Gesundheitswesen (Stakeholder, die die Dienste anbieten und diejenigen, die die Dienste nutzen) wählten aus den Ergebnissen der Delphi Befragung die wichtigsten Aspekte aus. In einem zweiten Workshop im selben Jahr bewerteten 50 Fachwissenschaftlerinnen und -wissenschaftler nochmals die erwähnten Standards und unterbreiteten zusätzliche Vorschläge. In zwei Arbeitstreffen stellten die Forschenden ein Set von Standards zusammen. 18 Akteurinnen und Akteure im Gesundheitswesen (Stakeholder) begutachteten das neue Set in einem weiteren Workshop. Interviews mit 3 erfahrenen Personen im Management des Gesundheitswesens wurden durchgeführt, um zusätzliche Aspekte, die bislang nur aus bisherigen Antworten interpretiert werden konnten, zu benennen. Ein erster Entwurf der überarbeiteten Standards wurde erstellt. Durch Interviews mit 3 Hebammen, die mit ähnlichen Standards arbeiten, flossen zusätzlich deren Erfahrungen ein. Nach einer zweiten Delphi Befragung mit 44 Teilnehmerinnen aus 13 Ländern zur Bewertung dieser neuen Liste von Standards begutachteten 12 Reviewerinnen und Reviewer aus Europa abschließend diese neue Liste.

\section{INHALTE}

\section{Midwifery Unit Standards}

Die insgesamt 29 Standards wurden zehn Themenbereichen zugeordnet:

1. Bio-psycho-soziales Modell der Betreuung: Unter diesem Thema fordert Standard 1 bspw. eine schriftlich festgehaltene und öffentlich zugängliche Philosophie für die Betreuung, die gemeinsame Werte und Überzeugungen des Personals aufzeigt.

2. Gleichheit, Vielfältigkeit und soziale Einbindung: Hier wird thematisiert, dass diejenigen, die im Geburtshaus arbeiten, Direktiven bezüglich Respekt, Diversität und Inklusion vereinbaren (Standard 2).

3. Interprofessionelle Zusammenarbeit: Einer der drei dort verorteten Standards besagt: Das gesamte geburtshilfliche Team teilt eine schriftliche Vereinbarung zu gegenseitigem Respekt und interdisziplinärem Arbeiten (Standard 3).

4. Wege für die Betreuung der Frauen: Einer der vier Standards betrifft eine klare Regelung zur Verlegung (Standard 9).

5. Personal und Arbeitsbelastung: Einer der zwei Standards fordert: Die Bewertung der Arbeitsbelastung sollte alle Aktivitäten in der Hebammeneinheit umfassen, nicht nur die intrapartale Betreuung und die Anzahl der Geburten (Standard 11).

6. Wissen, Skills und Training: Unter dieses Thema fallen drei Standards, darunter: Es ist schriftlich fixiert, welche Kenntnisse und Fähigkeiten Hebammen benötigen, um in einem Geburtshaus zu arbeiten (Standard 12).

7. Arbeitsumgebung und Ausstattung: Einer der fünf Standards zielt darauf ab, dass die (räumliche) Umgebung Mobilisierung und aktive Geburt für die Gebärende ermöglicht (Standard 16).

8. Autonomie und Verantwortlichkeit: Beide Standards in diesem Thema beschäftigen sich mit der Autonomie sowohl der Hebammen als auch der Gebärenden (Standards 20, 21).

9. Führung: Einer der drei unter diesem Thema geführten Standards fordert: Das Geburtshaus verfügt über eine konstante und gut erreichbare Leitung (Standard 22). 
10. Richtlinien: Unter diesem Thema werden fünf Standards gelistet, darunter: Das Geburtshaus engagiert sich für kontinuierliche Verbesserungen (Standard 27).

Quelle: Die gesamte 31 Seiten umfassende Liste der „Midwifery Unit Standards" ist zu finden unter www.midwiferyunitnetwork.org/mustandards [1].

\section{Ergebnisse}

Als Ergebnis der Literatursuche ist Folgendes festzuhalten: Zur wissenschaftlichen Evidenzbasierung von Vorgehensweisen, die zur Einrichtung, zum Management und zur Erhaltung und Qualitätssicherung von Geburtshäusern beitragen, gibt es Daten und Ergebnisse. Jedoch liegen noch längst nicht genügend Studien zu jedem dieser vier Aspekte vor. Erstmals wird eine Liste von Standards nicht von einem (kleinen und eher selbsternannten) Expertinnen- und Expertengremium auf Grund von Daten zum klinischen Outcome entworfen. Diese Liste wurde in einem Prozess erarbeitet, der erstens auf systematischer Literaturrecherche und zweitens auf den Erfahrungen der Praktiker bzw. Praktikerinnen sowie von Frauen, die Hebammenhilfe in Anspruch nehmen, basiert. Diese Ergebnisse wurden mit den Ansichten von Experten und Expertinnen kombiniert. Im Oktober 2019 haben diese „Midwifery Unit Standards“ die Befürwortung vom National Institute for Health and Care Excellence (NICE) erhalten.

\section{Schlussfolgerung der Autorinnen / Autoren}

Durch die Transparenz des Prozesses könnten die Ergebnisse auch außerhalb Großbritanniens von Interesse sein.

\section{Kommentar}

Der Erstellungsprozess dieser Standards ist vorbildhaft. Eine Schwäche der Arbeit liegt in der Tatsache, dass die meiste Evidenz aus englischsprachigen Ländern stammt. Zudem kommen die meisten Befragten aus Gesundheitssystemen, die mit dem deutschen System nicht verwandt sind. Daher bleiben beispielsweise für Aspekte wie die Zusammenarbeit des ambulanten mit dem stationären Bereich noch Standards unbearbeitet.

\section{Autorinnen / Autoren}

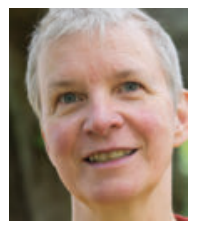

Dr. rer. medic. Christine

Loytved ist Hebamme und Gesundheitswissenschaftlerin. Sie arbeitet als Dozentin am Institut für Hebammen, Departement Gesundheit der Zürcher Hochschule für Angewandte Wissenschaften in Winterthur (Schweiz).
Korrespondenzadresse

E-Mail: loyt@zhaw.ch

Literatur

[1] Rocca-lhenacho L, Batinelli L, Thaels E, Rayment J, Newburn M, McCourt C. Midwifery Unit Standards. London: Midwifery Unit Network, City University of London; 2018. Im Internet: www.midwiferyunitnetwork.org/ mu-standards (abgerufen 30.04.2020)

Bibliografie

DOI https://doi.org/10.1055/a-1167-3526

Die Hebamme 2020; 33: 14-16

(c) Georg Thieme Verlag KG Stuttgart .

New York

ISSN 0932-8122 